\title{
OECD GUIDELINES FOR THE TESTING OF CHEMICALS
}

\author{
REVISED INTRODUCTION TO THE OECD GUIDELINES FOR TESTING OF \\ CHEMICALS, SECTION 3
}

\section{PART 1:PRINCIPLES AND STRATEGIES RELATED TO THE TESTING OF DEGRADATION OF ORGANIC CHEMICALS}

\section{DEGRADATION OF ORGANIC CHEMICALS}

\section{GENERAL}

1. Information on the degradability of organic chemicals may be used for hazard assessment or for risk assessment. Hazard assessment or risk in general, and aquatic hazard classification in particular, are normally based on data obtained in standardised tests for ready biodegradability, but results of tests simulating the biodegradation in water, aquatic sediment and soil may also be used for these purposes. Other types of test data that may be considered in an assessment of the potential environmental hazard or risk include sewage treatment plant (STP) simulation data, inherent biodegradability, anaerobic biodegradability, biodegradability in seawater and abiotic transformation.

2. In order to assess the environmental risk of particular chemicals, information allowing the estimation of its likely concentrations in the environment is necessary. Such an estimate must initially be based on knowledge of the likely use and disposal patterns of the chemical, its physical-chemical properties and the characteristics of the receiving environment

3. Degradation of organic chemicals in the environment influences exposure and, hence, it is a key parameter for estimating the risk of long-term adverse effects on biota. Degradation rates, or half-lives, are preferably determined in simulation biodegradation tests conducted under conditions that are realistic for the particular environmental compartment (e.g. STP, surface water, sediment or soil). Simulation tests aim at mimicking actual environmental conditions such as redox potential, $\mathrm{pH}$, temperature, microbial community, concentration of test substance and occurrence and concentration of other substrates.

4. These are important factors that determine the environmental degradation of organic chemicals in combination with the intrinsic properties of the chemical. The purpose of this introduction is to describe the principles of different types of degradation tests and to present guidance for the interpretation and use of degradability data.

\section{BIODEGRADATION IN WATER, SOILS AND SEDIMENTS}

\subsection{Introduction}

5. Because of the large number of chemicals that are being used in society an approach is required, which provides adequate knowledge for decision making as regards environmental protection, but which at the same time enables costs for testing to be kept to a minimum. Ideally, a system is required that allows preliminary screening of chemicals, using relatively simple tests of ultimate biodegradability, with the identification of those chemicals for which more detailed, and hence more costly, studies are needed. It is 
possible to organise the examination of the biodegradability of chemicals into a general testing strategy, consisting of tests of varying complexity, environmental realism and cost:

- First, aerobic biodegradability should be examined in a screening test for ready biodegradability;

- In the case of a negative result in a test for ready biodegradability, biodegradation of the chemical may be examined in a simulation test to obtain data to be used for assessing the biodegradation rate in the environment or in a biological STP. This would also be the case when a positive result for ready biodegradability has been obtained but when a more precise biodegradation half-life or $\mathrm{DT}_{50}$ is needed for risk assessment. Alternatively, or as a supplement, a screening test for inherent biodegradability may be conducted for generation of data describing the potential biodegradability under optimised aerobic conditions, such as those which may potentially occur in STPs at long sludge ages;

- In addition, potential biodegradability under anoxic conditions may be examined in a screening test for anaerobic biodegradability.

\subsection{Definitions}

\section{Ready biodegradability tests}

6. Stringent screening tests, conducted under aerobic conditions, in which a high concentration of the test substance (in the range of 2 to $100 \mathrm{mg} / \mathrm{L}$ ) is used and biodegradation is measured by non-specific parameters like Dissolved Organic Carbon (DOC), Biochemical Oxygen Demand (BOD) and $\mathrm{CO}_{2}$ production. Domestic sewage, activated sludge or secondary effluent is the typical source of microorganisms (inoculum) in tests for ready biodegradability. The inoculum should not have been preadapted to degradation of the test substance by previous exposure to the test substance or structurally related chemicals. A positive result in a test for ready biodegradability can be considered as indicative of rapid and ultimate degradation ${ }^{1}$ in most environments including biological STPs.

7. A chemical attaining the pass level in these tests at a certain rate after termination of the lag phase may be classified as "readily biodegradable". The pass level depends on the analytical parameter measured.

\section{Simulation tests}

8. Aerobic and anaerobic tests that provide data for biodegradation under specified environmentally relevant conditions. These tests simulate the degradation in a specific environment by use of indigenous biomass, media, relevant solids (i.e. soil, sediment, activated sludge or other surfaces) to allow sorption of the chemical, and a typical temperature which represents the particular environment. A low concentration of test substance is used in tests designed to determine the biodegradation rate constant whereas higher concentrations are normally used for identification and quantification of major transformation products for analytical reasons.

9. A low concentration of chemical in these types of tests refers to a concentration (e.g. less than 1 $\mu \mathrm{g} / \mathrm{L}$ to $100 \mu \mathrm{g} / \mathrm{L}$ ), which is low enough to ensure that the biodegradation kinetics obtained in the test reflect those expected in the environment being simulated. Biodegradation is measured either by radiolabelling techniques or by specific chemical analyses. Tests of these types may be subdivided

\footnotetext{
${ }^{1}$ Ultimate degradation is the degradation of the substance to $\mathrm{CO}_{2}$, biomass, $\mathrm{H}_{2} \mathrm{O}$ and other inorganic substances like $\mathrm{NH}_{3}$
} 
according to the environment that they are designed to simulate, e.g.: a) soil, b) aquatic sediments, c) surface water and d) STPs.

\section{Inherent biodegradability tests}

10. Aerobic tests that possess a high capacity for degradation to take place, and in which biodegradation rate or extent is measured. The test procedures allow prolonged exposure of the test substance to microorganisms and a low ratio of test substance to biomass, which offers a better chance to obtain a positive result compared to tests for ready biodegradability. Some of these tests may be conducted using microorganisms that have previously been exposed to the test substance, which frequently results in adaptation leading to a significant increase of the degradation rate.

11. A substance yielding a positive result in a test of this type may be classified as "inherently biodegradable", which, preferably, should be qualified by one of the terms "with pre-adaptation" or "without pre-adaptation" as appropriate. Because of the favourable conditions employed in these tests, rapid biodegradation in the environment of inherently biodegradable chemicals cannot generally be assumed.

\section{Anaerobic biodegradability screening tests}

12. Screening tests, conducted under anoxic conditions, in which a high concentration of the test substance $(\mathrm{mg} / \mathrm{L})$ is used and biodegradation is measured by non-specific parameters like total inorganic carbon (TIC), $\mathrm{CO}_{2}$ and $\mathrm{CH}_{4}$ production. These tests are used for the evaluation of potential anaerobic biodegradability in an anaerobic digester at a given range of concentration of microorganisms.

\subsection{Ready biodegradability tests}

13. Ready biodegradability tests must be designed so that positive results are unequivocal. Given a positive result in a test of ready biodegradability, it may be assumed that the chemical will undergo rapid and ultimate biodegradation in the environment. In such cases, no further investigation of the biodegradability of the chemical, or of the possible environmental effects of transformation products, is normally required. However, the fact that the chemical is found to be readily biodegradable does not preclude concern about biodegradation rate constants and the transformation products in cases of high influx into a receiving environment. Realising that ready biodegradability tests may sometime fail because of the stringent test conditions, consistent positive test results from test(s) should generally supersede negative test results. However, when conflicting test results are reported, it is recommended to check the origin of the inoculum in order to check whether or not differences in the adaptation of the inoculum may be the reason.

14. When the risk of adverse effects cannot be excluded, as is the case for some high production volume chemicals, it is recommended that the biodegradation rate of the parent substance in a relevant simulation test be determined. If necessary, a risk assessment including the parent substance and possible major transformation products may be performed.

15. A negative result in a test for ready biodegradability does not necessarily mean that the chemical will not be degraded under relevant environmental conditions, but it means that the next level of testing, i.e. either a simulation test or an inherent biodegradability test, should be considered.

16. The OECD tests which can be used to determine the ready biodegradability of organic chemicals include the six test methods described in the OECD Test Guidelines No. 301 A-F: DOC Die-Away Test (TG $301 \mathrm{~A}$ ), $\mathrm{CO}_{2}$ Evolution Test (TG $301 \mathrm{~B}$ ), Modified MITI Test (I) (TG $301 \mathrm{C}$ ), Closed Bottle Test (TG 301 D), Modified OECD Screening Test (TG 301 E) and Manometric Respirometry Test (TG 301 F). The 
following pass levels of biodegradation, obtained within 28 days, may be regarded as evidence of ready biodegradability: 70\% DOC removal (TG $301 \mathrm{~A}$ and TG $301 \mathrm{E}$ ); $60 \%$ theoretical carbon dioxide $\left(\mathrm{ThCO}_{2}\right)$ (TG $301 \mathrm{~B}$ ); 60\% theoretical oxygen demand (ThOD) (TG 301 C, TG $301 \mathrm{D}$ and TG $301 \mathrm{~F}$ ). Suggestions to decrease the pass level of the respirometric tests of TG301 from $60 \%$ to $50 \%$ have been put forward by contract laboratories and in the literature. Such a change has, however, not yet taken place.

17. These pass levels have to be reached in a 10-day window within the 28-day period of the test. The 10-day window does not apply to TG $301 \mathrm{C}$. The 10-day window begins when the degree of biodegradation has reached $10 \%$ DOC removal, ThOD or $\mathrm{ThCO}_{2}$ and must end before or at day 28 of the test. The pass levels of either $60 \%$ ThOD (or $\mathrm{ThCO}_{2}$ ) or $70 \%$ DOC removal practically represent complete ultimate degradation of the test substance as the remaining fraction of $30-40 \%$ of the test substance is assumed to be assimilated by the biomass or present as products of biosynthesis.

18. Another test for ready biodegradability, which represents an alternative to the $\mathrm{CO}_{2}$ Evolution Test (TG $301 \mathrm{~B}$ ), is the Headspace Test (Ready Biodegradability $-\mathrm{CO}_{2}$ in sealed vessels; TG 310). In this test, the $\mathrm{CO}_{2}$ evolution resulting from the ultimate aerobic biodegradation of the test substance is determined by measuring the inorganic carbon (IC) produced in sealed test bottles, and the pass level has been defined as $60 \%$ of theoretical maximum IC production (ThIC).

19. As all of the above-mentioned methods pertain to conditions in fresh waters, screening test procedures suitable for marine environments have been described: The OECD TG 306 on Biodegradability in Seawater includes seawater variants of the Closed Bottle Test (TG 301 D) and of the Modified OECD Screening Test (TG $301 \mathrm{E}$ ). Degradation of organic chemicals in seawater has generally been found to be slower than that in freshwater, activated sludge and sewage effluent, and, therefore, a positive result obtained during 28 (Closed Bottle Method) or 60 days (Shake Flask Method) in the biodegradability in Seawater test can be regarded as evidence of a chemical's potential for biodegradation in the marine environment. For example, a result of $>20 \%$ ThOD or DOC removal is indicative of potential for primary biodegradation in the marine environment, whereas a result of $>60 \%$ ThOD or $70 \%$ DOC removals is indicative of potential for ultimate biodegradation in the marine environment.

20. The above test guidelines are similar in several respects: In all the tests, the test substance providing the sole source of organic carbon (except for carbon associated with the biomass) is diluted in a test medium containing a relatively low concentration of biomass. In all the tests, a non-specific analytical method is used to follow the course of biodegradation. This has the advantage that the methods are applicable to a wide variety of organic substances and there is no need to develop specific analytical procedures. Since these methods also respond to any biodegradation residues or transformation products, an indication of the extent of ultimate biodegradation is provided.

21. The standardised test duration is 28 days although tests may be prolonged beyond 28 days if the biodegradation has started but has not yet reached a plateau. However, only the extent of biodegradation achieved within 28 days should be used for the evaluation of ready biodegradability but degradation after 28 days would allow the test substance to be classified as inherently biodegradable (see paragraph 36 ).

22. It has been recognised that standardisation of the inoculum might also improve the comparability of the methods. However, it was concluded that this is not possible without significantly reducing, at the same time, the number of species present in the test system. A mixed inoculum is therefore recommended to ensure the presence of a variety of degrading organisms in the tests. In view of the stringent requirements to these tests, it was also decided that pre-exposure (i.e. pre-adaptation) of the inoculum to the test substance should not be allowed. If pre-exposed inoculum was used, the test is per definition no longer a test for ready biodegradability, and a positive result may then be used to classify the test substance as "inherently biodegradable with pre-adaptation" (see paragraph 11). An OECD inter-laboratory 
comparison exercise (ring test) (1) took place in 1988 in order to ensure the practicability and validity of the tests.

\section{$2.4 \quad$ Simulation tests}

23. Simulation tests aim at assessing the rate and extent of biodegradation in a laboratory system designed to represent either the aerobic treatment stage of STP or environmental compartments, such as fresh or marine surface water.

\section{Sewage treatment}

24. The fate of chemicals in STPs can be studied in the laboratory by using the Simulation Test Aerobic Sewage Treatment: Activated Sludge Units (TG 303 A) and Biofilms (TG 303 B). The removal of the test substance is determined by monitoring the changes in DOC and/or Chemical Oxygen Demand (COD). The basic test procedures (TG $303 \mathrm{~A}$ and TG $303 \mathrm{~B}$ ) recommend addition of the test substance at a concentration of DOC between $10 \mathrm{mg} / \mathrm{L}$ and $20 \mathrm{mg} / \mathrm{L}$. However, many chemicals are normally present at very low concentrations, even in waste water, and procedures for testing the biodegradation at suitably low concentrations $(<100 \mu \mathrm{g} / \mathrm{L})$ are presented in Annex 7 to the TG $303 \mathrm{~A}$.

25. No specific pass levels have been defined for the elimination of chemicals in aerobic sewage treatment simulation tests. It is noted that such a level would have to be related to the specific operating conditions and plant design. The test results may be used to estimate the removal in STPs and the resulting effluent concentrations for prediction of the concentration in the treatment plant and the receiving aquatic environment.

26. Monod kinetics ${ }^{2}$ (originally proposed for pure cultures and single substrate systems only) may be used for description of the degradation of a substance when it is assumed that growth is a continuous process, and that the biomass is produced during utilisation of the test substance. It follows from Monod kinetics that the outlet concentration is independent of the inlet concentration, whereas this is not the case where the test substance serves as a secondary substrate for the degrading biomass. The presence of the test substance as a secondary substrate represents a low concentration scenario, which implies that the degradation rate may be expressed by pseudo-first order $^{3}$ or first order kinetics ${ }^{4}$ (2)(3).

\section{Soil, sediment and water}

27. The following tests can be used to simulate the biodegradation of organic chemicals under environmentally realistic conditions in soil, sediment or surface water: Aerobic and Anaerobic Transformation in Soil (TG 307); Aerobic and Anaerobic Transformation in Aquatic Sediment Systems (TG 308); and Aerobic Mineralisation in Surface Water - Simulation Biodegradation Test (TG 309).

\footnotetext{
${ }^{2}$ The rate of degradation of the test substance in a laboratory study where the substance is the sole source of carbon and energy may be described by: $-\mathrm{dS} / \mathrm{dt}=\left[\left(\mu_{\max } / \mathrm{Y}\right) \cdot \mathrm{B} \cdot \mathrm{S}\right] /\left[\mathrm{K}_{\mathrm{s}}+\mathrm{S}\right]$; where: $-\mathrm{dS} / \mathrm{dt}$ is the degradation rate, $\mu_{\max }$ is the maximum specific growth rate, $\mathrm{Y}$ is the yield coefficient, $\mathrm{B}$ is the biomass concentration, $\mathrm{K}_{\mathrm{s}}$ is the half saturation constant, and $\mathrm{S}$ is the concentration of the test substance. where $\mathrm{k}_{1}$ is the first order rate constant. It is assumed that the concentration of biomass (B) is constant during the experiment.

${ }^{3}$ The rate of degradation is proportional to the concentration of the test substance and biomass, i.e.: $-\mathrm{dS} / \mathrm{dt}=\mathrm{k}_{1} \cdot \mathrm{S} \cdot \mathrm{B}$;

${ }^{4}$ First order kinetics, i.e.- $\mathrm{dS} / \mathrm{dt}=\mathrm{k}_{1} \cdot \mathrm{S}$, may be used when the degradation of the test substance is independent of the concentration of biomass.
} 
28. Aerated soils are aerobic, whereas water-saturated or water-logged soils are frequently dominated by anaerobic conditions. The surface layer of aquatic sediments can be either aerobic or anaerobic, whereas the deeper sediment is usually anaerobic. These conditions in soil or sediment may be simulated by using aerobic or anaerobic tests described in the test guidelines (TG 307 and TG 308).

29. Generally, a low concentration of the test substance is used in tests designed to determine biodegradation. A low concentration in these types of tests means a concentration (e.g. from $1 \mu \mathrm{g} / \mathrm{L}$ to 100 $\mu \mathrm{g} / \mathrm{L}$ in TG 309), which is low enough to ensure that the biodegradation kinetics (first order or pseudo-first order) obtained in the test reflect those expected in the environment.

30. The temperature dependence of the kinetic constants must be considered. It is recommended to perform the test at a temperature characteristic of the environment which is simulated.

31. When using radiolabelled chemicals, the label should be located in the most recalcitrant part of the molecule when total mineralization is assessed. If the most stable structure does not include the functional or environmentally relevant part of the molecule, it may be appropriate to use a test chemical with a different labelling.

32. Measuring disappearance of the parent compound by chemical analysis does not imply mineralisation. Simulation tests are especially useful if it is known from other tests that the test substance can be mineralised and that the degradation, which is measured, covers the rate determining process.

33. The results of simulation tests may include:

- First order or pseudo-first order rate constant;

- Degradation half-life ${ }^{5}$ or $\mathrm{DT}_{50}{ }^{6}$;

- Half-saturation constant;

- Maximum specific growth rate;

- Fraction of mineralised label, and, if specific analyses are used, the final level of primary degradation;

- Mass balance during and at the end of the study;

- Identification and concentration of major transformation products, where appropriate;

- A proposed pathway of transformation, where appropriate.

For a complete overview of the result parameters in relation to the simulation degradation test guidelines please refer to these guidelines (TG 303, TG 304, TG 307, TG 308 and TG 309).

\subsection{Inherent biodegradability tests}

34. Using favourable conditions, the tests of inherent biodegradability have been designed to assess whether the chemical has any potential for biodegradation under aerobic conditions. The tests for inherent biodegradability vary in their degradation capacity (see paragraph 35), and the differences in test conditions should be considered if the results are used as an indication of potential biodegradation or environmental persistence. Inherent biodegradability can be measured by specific analysis (primary biodegradation) or by non-specific analysis (ultimate biodegradation).

\footnotetext{
${ }^{5}$ Half-life $\left(\mathrm{t}_{0.5}(\mathrm{~d})\right)$ is characteristic of the rate of a first-order reaction and corresponds to the time interval for the concentration to decrease by a factor of two.

${ }^{6} \mathrm{DT}_{50}$ (Disappearance Time 50) is the time within which the concentration of the test substance is reduced by $50 \%$; it is different from the half-life $\mathrm{t}_{0.5}$ when transformation does not follow first order kinetics.
} 
35. The tests that can be used to determine the inherent biodegradability of organic chemicals include three methods described in the OECD Test Guidelines No. 302 A-C: Modified SCAS Test (TG 302 A), Zahn-Wellens/EMPA Test (TG 302 B) and Modified MITI Test (II) (TG 302 C) The biodegradation capacity in these tests increases in the order TG $302 \mathrm{C}<\mathrm{TG} 302 \mathrm{~B}<\mathrm{TG} 302 \mathrm{~A}$.

36. Since inherent biodegradability can be considered to be a specific property of a chemical, it is not necessary to define limits on test duration or biodegradation rates. Biodegradation above $20 \%$ of theoretical (measured as BOD, DOC removal or COD) may be regarded as evidence of inherent, primary biodegradability, whereas biodegradation above $70 \%$ of theoretical (measured as BOD, DOC removal or COD) may be regarded as evidence of inherent, ultimate biodegradability. When results of ready biodegradability tests indicate that the pass level criterion is almost fulfilled (i.e. ThOD or DOC slightly below $60 \%$ or $70 \%$ respectively) such results can be used to indicate inherent biodegradability. This is also the case when the pass level criterion is fulfilled but the 10-day window criterion is not. Such application of ready biodegradability tests, which may include their incubation beyond 28 days, may in some cases eliminate the need for additional testing of biodegradability in inherent or simulation tests.

37. When the results indicate that inherent, ultimate biodegradability does occur, it indicates that the substance has a potential for degradation under favourable conditions, e.g. in well-operated STPs. When a negative result is obtained in a test of inherent biodegradability, it may lead to a preliminary conclusion of environmental persistence and to an evaluation of potential adverse effects of transformation products. An alternative is to examine ultimate biodegradation at environmentally realistic low concentrations of the chemical in a simulation test.

\subsection{Anaerobic biodegradability screening tests}

38. The potential anaerobic biodegradability of organic chemicals can be determined by using the test: Anaerobic Biodegradability of Organic Compounds in Digested Sludge/By Measurement of Gas Production (draft TG 311). The test substance, which is the sole added organic carbon in the test, is exposed to diluted anaerobically digested sludge of a relatively low concentration. Biodegradability of the test substance is followed by measurements of the increase in headspace pressure resulting from the evolution of $\mathrm{CO}_{2}, \mathrm{CH}_{4}$ and TIC.

39. A test duration of 60 days is recommended but the test may be prolonged beyond 60 days or terminated earlier if the biodegradation has reached a plateau, indicating a sufficient degree of biodegradation ( $>60 \%$ of theoretical gas production). No formal decisions on criteria for anaerobic biodegradability have been made but, tentatively, the lowest value $(60 \%)$ for ready aerobic biodegradability $\left(60 \%\right.$ ThOD or $\left.60 \% \mathrm{ThCO}_{2}\right)$ has been adopted.

40. The draft TG 311 is designed to assess the ultimate anaerobic biodegradability of organic chemicals in heated digesters for anaerobic sludge treatment. The test is, therefore, not necessarily applicable to anoxic environmental compartments such as anoxic sediments and soils.

\subsection{Interpretation of results}

\section{Ready biodegradability tests}

41. In order to interpret the results of a test, the full biodegradation curve should be considered so that the duration of the lag phase, slope and plateau level can be identified. The duration of 28 days in the ready biodegradability tests was defined in order to allow for sufficient time for the microorganisms to adapt to the chemical (lag phase) by an increase in the number of active degrading microorganisms that results in detectable degradation. 
42. While the test duration of 28 days allows time for adaptation, chemicals that degrade slowly after a short lag phase should not be considered as readily biodegradable in tests employing the 10-day window, if the rate is too slow for fulfilling the 10-day window or an equivalent rate criterion. It is, therefore, required that the pass level of ready biodegradability is reached within 10 days of the start of biodegradation (10-day window). Biodegradation should be considered to have started when it exceeds the $10 \%$ level.

43. Although these tests are intended for pure chemicals, it is sometimes relevant to examine the ready biodegradability of mixtures of structurally similar chemicals like oils and surface-active substances (surfactants). Such substances often occur as mixtures of constituents with different chain-lengths, degree and/or site of branching or stereo-isomers, even in their most purified commercial forms. Testing of each individual component may be costly and impractical. If a test on the mixture is performed and it is anticipated that a sequential biodegradation of the individual structures is taking place, then the 10-day window should not be applied to interpret the results of the test. A case by case evaluation should however take place on whether a biodegradability test on such a complex mixture would give valuable information regarding the biodegradability of the mixture as such (i.e. regarding the degradability of all the constituents) or whether instead an investigation of the degradability of carefully selected individual components of the mixture is required.

44. It should be noted that such mixtures are here regarded as technical materials of similar types of chemicals (e.g. homologues of surfactants composed of fatty alcohols of varying chain length, or poly(oxyalkylene) polyol materials having defined molecular weight distributions). Tests for ready biodegradability are not generally applicable for complex mixtures containing different types of chemicals.

45. The results of a ready biodegradability test may be used for aquatic hazard classification of chemicals. According to the principles described in the "Harmonised Integrated Classification System for Human Health and Environmental Hazards of Chemical Substances and Mixtures" (4), a positive result in one of the OECD tests for ready biodegradability can be considered as indicative of rapid degradation in most environments. Positive results obtained by the TG 306, which is more suitable for marine environments, can also be considered as evidence of rapid degradability.

46. Results from ready biodegradability tests may be used for assessment of biodegradation in a specific environmental compartment, when no data from tests simulating the conditions in that compartment are available (5)(6). First order rate constants may be derived with the purpose of modelling the biodegradation in STPs, surface water, sediment and soil by using pragmatic principles (see examples in paragraph 47 and 48 ).

47. For example, the European Commission Technical Guidance Document on Risk Assessment (5) prescribes that a rate constant $(\mathrm{k})$ of 1.0 hour $^{-1}$, and a half-life of 0.69 hours, may be assigned to readily biodegradable chemicals (fulfilling the pass level and the 10-day window) in models for estimating the elimination of chemicals in STPs (STP models). A lower rate constant of 0.3 hour $^{-1}$, equivalent to a halflife of 2.3 hours, may be used in STP models, if a chemical reaches the pass level during the 28-day period, but fails the 10-day window (5).

48. The same objective is being addressed in a U.S. Environmental Protection Agency guidance document describing the use of biodegradability data for multimedia models and STP models (6). This document describes how results of ready biodegradability tests may be used to derive activated sludge halflives as indicated below:

- Readily degradable chemicals:

- Chemicals attaining $\geq 40 \%$ degradation:

- Chemicals attaining $\geq 20$ but $<40 \%$ degradation: 10 hours $\left(\mathrm{k}=0.069\right.$ hour $\left.^{-1}\right)$. 
49. If the biodegradability of the chemical does not reach the pass level, it is recommended to examine whether it was inhibitory to microbial activity at the concentration used in the test. If the test substance was inhibitory, it may be re-tested at low, non-inhibitory concentrations in a relevant simulation test (TG 303, TG 307, TG 308 or TG 309). Re-testing in a modified ready biodegradability test at a much lower concentration (i.e. more than 10 times lower than prescribed) cannot generally be recommended as suitable simulation test methods are available (4)(5).

\section{Simulation tests}

50. A chemical that fails to meet the criteria for ready biodegradability, or even inherent biodegradability, may be rapidly degradable when present at low concentrations in the environment. Simulation tests may be used to examine the biodegradation of organic chemicals in STPs (TG 303 A and TG 303 B), soil (TG 307), aquatic sediment (TG 308) and surface water (TG 309). If it can be demonstrated that the chemical is ultimately degraded by more than $70 \%$ in 28 days under realistic conditions in the aquatic environment (e.g. by using TG 308 or TG 309), then the definition of "rapid degradability" in relation to aquatic hazard classification will have been met (4).

51. The results of a simulation test may show a rapid transformation of the parent compound, whereas the ultimate degradation, measured by e.g. $\mathrm{CO}_{2}$ gas production, is limited due to the formation of recalcitrant transformation products. It is, therefore, necessary to distinguish between primary and ultimate biodegradation, when the rate and extent of degradation are calculated.

52. If first order kinetics are assumed, which is reasonable at the low substance concentrations prevailing in most aquatic environments, the requirement for "rapid degradability" in relation to aquatic hazard classification will be satisfied, when a chemical is ultimately degraded in a simulation test with a half-life of less than 16 days (4). Results of aquatic simulation tests (e.g. TG 308, TG 309) may be used directly for aquatic hazard classification purposes, when realistic environmental conditions are simulated, i.e. when:

- Substance concentration which is realistic for the general aquatic environment (often in the low $\mu \mathrm{g} / \mathrm{L}$ or $\mu \mathrm{g} / \mathrm{kg}$ range);

- Inoculum is from a relevant aquatic environment;

- Inoculum concentration is realistic (e.g. $10^{3}-10^{6}$ cells $/ \mathrm{mL}$ in surface water);

- Temperature is realistic (e.g. $5^{\circ} \mathrm{C}$ to $25^{\circ} \mathrm{C}$ );

- Ultimate degradation is determined (i.e. determination of the mineralisation rate or the individual degradation rates of all relevant transformation products).

53. If no data are available from aquatic simulation or screening tests, the degradation rate of a chemical in surface water may be estimated by using results of a simulation test for degradation in soil (e.g. TG 307). In relation to aquatic hazard classification, a chemical may be considered rapidly degradable in the aquatic environment, if it is ultimately degraded in soil with a half-life of less than 16 days provided that no pre-exposure has taken place and that a realistic concentration of the test chemical has been employed (see reference (4), Annex 2, Chapter 4). In relation to risk assessment similar approaches have been proposed for extrapolation of results from biodegradation tests to rate constants in surface water, sediment and soil (5)

54. Whenever possible, assessment of biodegradation in the environment should be based on results from tests simulating the conditions in the relevant environmental compartment. Degradation half-life and kinetic constants determined in a simulation test should be corrected to the average outdoor temperature or it should be documented that the difference between test temperature and outdoor temperature is negligible. 
55. It should be noted that results of a simulation test should only be extrapolated to degradation in the real environment, if the concentrations used in the test were in the same order of magnitude as the concentrations expected in the environment.

56. Man-made organic chemicals will normally be present at low concentrations (i.e. low $\mu \mathrm{g} / \mathrm{L}$ level) in the environment compared to the total mass of biodegradable carbon substrates. This implies that the anticipated biodegradation kinetics are first order ("non-growth" kinetics). If a higher concentration is used in a test (e.g. to examine transformation products), biodegradation of the chemical will frequently support growth of the degrading microorganisms.

57. Degradation kinetics in soil or sediments may often deviate from first order kinetics because sorption/desorption processes take place simultaneously with degradation processes. In such cases expert judgement is required for estimating a degradation half-life or half-lives for various sub-compartments (7).

58. When results from more than one simulation test are available, a suitable half-life or $\mathrm{DT}_{50}$ in the higher end of the observed range should be used for estimating environmental degradation, taking into account the realism, relevance, quality and documentation of the studies in relation to the environmental conditions (5).

\section{Inherent biodegradability}

59. Inherent biodegradability tests are used to assess whether a chemical has any potential for biodegradation. The European Commission Technical Guidance Document (5) proposes that results of the Zahn-Wellens/EMPA Test (TG 302 B) and the Modified MITI Test (II) (TG 302 C) may be used for extrapolation to a rate constant in models for estimation of the elimination of chemicals in STPs (5). However, this extrapolation is only allowed, if the inherent biodegradability tests fulfil specific criteria ${ }^{7}$.

60. Also the approach of the U.S. Environmental Protection Agency and Environment Canada to derive input data for multimedia models and STP models (6)(8)(9) includes principles for extrapolation of inherent biodegradability test results to activated sludge half-lives in STP models and surface water. A negative result in tests for inherent biodegradability may lead to a preliminary conclusion of environmental persistence, but it should not be regarded as a definitive evidence of persistence as the high concentration of the test substance may impede ultimate biodegradability by inhibiting the degrading microorganisms.

61. If the microorganisms in the test are inhibited by the test substance, it is recommended that ultimate biodegradability of the chemical be examined in a simulation test by using a realistic source of inoculum, realistic temperature and a realistic low concentration of test substance.

\footnotetext{
${ }^{7}$ The pass level of $70 \%$ degradation in the Zahn-Wellens/EMPA Test must be reached within 7 days, including the lag-phase and the log-phase, the log-phase should be no longer than 3 days, and the percentage removal in the test before biodegradation occurs should be below 15\%. The pass level of 70\% in the Modified MITI Test (II) must be reached within 14 days, including the lag-phase and the log-phase, and the log-phase should be no longer than 3 days (5).
}

Lag-phase: During the lag-phase, growth of specific microorganisms may be exponential, but due to their small number, it takes several doubling times before biodegradation becomes detectable above the background value of the inoculum.

Log-phase: During the log-phase, exponential growth of specific microorganisms continues and the maximum growth rate $\mu_{\max }$ remains constant; the log-phase ends, when depletion of the parent substance leads to a decrease of the growth rate below $\mu_{\max }$. 


\section{Potential anaerobic biodegradability}

62. The screening test for potential anaerobic biodegradability (TG 311, Anaerobic Biodegradability of Organic Compounds in Digested Sludge / By Measurement of Gas Production) is performed at a high incubation temperature $\left(35^{\circ} \mathrm{C}\right)$, which approximates the temperature in heated digesters for anaerobic sludge treatment. This temperature favours anaerobic biodegradation of chemicals with low or moderate toxicity to anaerobic bacteria. On the other hand, the high concentration of test substance may inhibit the ultimate biodegradation of toxic chemicals.

63. The test temperature implies that the results obtained are not necessarily representative of other anoxic environments such as aquatic sediments. If the test substance was inhibitory in the screening test, it may be re-tested at low non-inhibitory concentrations in a relevant simulation test, e.g., TG 308, which can be conducted under strictly anaerobic conditions.

\section{ABIOTIC TRANSFORMATION}

\subsection{Introduction}

64. Chemicals in aquatic environments, soil and air may be transformed by abiotic processes such as hydrolysis, oxidation and photolysis. Abiotic transformation can be an important step in the pathway for degradation of man-made chemicals in the environment. In the atmosphere, abiotic transformation (oxidation by hydroxyl radicals) is the most important process for the complete destruction of air-borne chemicals. Although abiotic transformation in water, sediments and soil in itself usually is only primary degradation, the products formed by such abiotic processes may be biodegraded further by microorganisms.

65. Generally, the most important processes for the degradation of most chemicals in the aquatic environment are biodegradation or combined degradation by hydrolysis and subsequent biodegradation. In aquatic systems, sediments and soil even slow hydrolysis or biodegradation is likely to be more important than phototransformation, because of the limited possibility for exposure to sunlight in aquatic systems. Dependent of environmental conditions, time of the year and latitude, phototransformation may be an important step in the initial transformation of some chemicals, which may lead to biodegradable transformation products.

\subsection{Hydrolysis}

66. Abiotic hydrolytic transformation of chemicals in aquatic systems may be examined at $\mathrm{pH}$ values normally found in the environment $(\mathrm{pH} 4-9)$ by use of the guideline: Hydrolysis as a Function of $\mathrm{pH}$ (revised TG 111, Adopted 13 April, 2004). This method is generally applicable to chemical substances $\left({ }^{14} \mathrm{C}\right.$-labelled or non-labelled) for which an analytical method with sufficient accuracy and sensitivity is available. The results of a test of hydrolysis may include:

- Repeatability and sensitivity of the analytical methods;

- Recoveries;

- Mass balance during and at the end of the study (when ${ }^{14} \mathrm{C}$-labelled test substance is used);

- Half-life or $\mathrm{DT}_{50}$.

67. Most hydrolysis reactions follow apparent first order reaction rates and, therefore, half-lives are independent of the concentration. This usually permits the extrapolation of laboratory results determined at $10^{-2}$ to $10^{-3} \mathrm{M}$ to environmental conditions $\left(\leq 10^{-6} \mathrm{M}\right)(10)$. 


\subsection{Phototransformation}

68. The potential effects of solar irradiation on the fate of chemicals in surface water and soil, respectively, may be examined by use of the guidelines: Phototransformation of Chemicals in Water Direct and Indirect Photolysis (draft August 2000) and Phototransformation of Chemicals on Soil Surfaces (draft January 2002).

69. The rate of photolysis of a chemical in the environment depends on several factors. These include the attenuation of solar light in natural water bodies and the intensity of solar radiation, the latter of which is itself dependent on factors such as latitude and season. Any data on half-lives or $\mathrm{DT}_{50}, \mathrm{DT}_{75}$ and $\mathrm{DT}_{90}$ values should be reported along with calculations associated with these data, and the results of any outdoor experiments, if the latter have been conducted. Where possible, information on transformation products should be provided as well.

\section{LITERATURE}

(1) OECD (1998). OECD Ring-test of Methods for determining Ready Biodegradability.

(2) Berg, U.T. and N. Nyholm (1996). Biodegradability simulation studies in semicontinuous activated sludge reactors with low ( $\mu \mathrm{g} / \mathrm{l}$ range) and standard (ppm range) chemical concentrations. Chemosphere 33, 711-735.

(3) Nyholm, N., F. Ingerslev, U.T. Berg, J.P. Pedersen and H. Frimer-Larsen (1996). Estimation of kinetic rate constants for biodegradation of chemicals in activated sludge wastewater treatment plants using short-term batch experiments and $\mu \mathrm{g} / \mathrm{l}$ range spiked concentrations. Chemosphere 33 , 851-864.

(4) OECD (2001). Harmonised Integrated Classification System for Human Health and Environmental Hazards of Chemical Substances and Mixtures. OECD Series on Testing and Assessment. No. 33.

(5) European Commission (2003). Technical Guidance Document on Risk Assessment in Support of Commission Directive 93/67/EEC on Risk Assessment for new Notified Substances and Commission Regulation (EC) No. 1488/94 on Risk Assessment for Existing Substances and Directive 98/8/EC of the European Parliament and of the Council Concerning the Placing of Biocidal Products on the Market.

(6) U.S. Environmental Protection Agency (2000). Interim Guidance for Using Ready and Inherent Biodegradability Tests to Derive Input Data for Multimedia Models and Wastewater Treatment Plants (WWT) Models.

Available on the following website: http://www.epa.gov/oppt/exposure/docs/halflife.htm

(7) Wolt J.D, H.P.Nelson Jr, C.B. Cleveland, and I.J. van Wesenbeeck (2001): Biodegradation kinetics for pesticide exposure assessment, Rev. Environ. Contam. Toxicol., 169, 123-164.

(8) BEC (Bonnell Environmental Consulting) 2001, Conducting the Multi-Media Exposure Assessment of New Substances in Canada. Final report. Prepared under contract for the New Substances Division, Environment Canada; Ottawa, Canada. July.

(9) Webster, E., Mackay D., Wania, F, Arnot, J., Gobas, F., Gouin, T., Hubbarde, J. and M. Bonnell. 2005, Development and Application of Models of Chemical Fate in Canada. Final Report to 
Environment Canada Contribution Agreement 2004-2005. Canadian Environmental Modelling Network, Peterborough, Ontario. March.

(10) Mabey, W. and Mill, T. (1978). Critical review of hydrolysis of organic compounds in water under environmental conditions. J. Phys. Chem. Ref. Data 7, 383-415. 
PART 2:BIOACCUMULATION AND BEHAVIOR IN SOILS AND SEDIMENTS

NOTE: RELATIVELY FEW NEW OR REVISED OECD GUIDELINES FOR TESTING HAVE BEEN ISSUED CONCERNING ACCUMULATION AND ENVIRONMENTAL FATE IN SOIL AND SEDIMENT SINCE THIS ANNEX WAS ORIGINALLY APPROVED. NEVERTHELESS THE TEXT \begin{tabular}{lllllllll}
\hline BELOW IS CURRENTLY UNDER & REVISION & (AND & A & DETAILED & REVIEW & PAPER
\end{tabular} CONCERNING BIOACCUMULATION IS ALSO BEING DRAFTED FOR MAKING PRIORITIES FOR FUTURE TEST GUIDELINE DEVELOPMENT).

\section{BIOACCUMULATION}

\section{GENERAL}

1. The extent of bioaccumulation of a chemical at a given time is the combined result of the competing processes of uptake, distribution, transformation and excretion. If the chemical is available for uptake by the organism over a period of time, the four interconnected processes result in a dynamic equilibrium (apparent plateau or steady-state) which is characterised by a constant ration of the chemical in the test organism and in the ambient medium (e.g. water) or food. This ratio is the mathematical quantification of bioaccumulation.

2. It can be assumed that a chemical which does not readily undergo metabolic transformation may express its potential for bioaccumulation, though to a different degree, in many species, provided it is present in the ambient environments, and/or food of these species, for a sufficiently long period of time.

3. It has been proven experimentally convenient in the study of bioaccumulation processes to consider the two possible routes of uptake separately: direct bioaccumulation from the ambient medium and indirect bioaccumulation via the food chain.

4. On the basis of the knowledge gathered in the past few years, direst bioaccumulation unequivocally predominates over indirect bioaccumulation in the aquatic environment, whereas indirect bioaccumulation is the dominant mechanism effective in terrestrial animals.

5. A critical review of the various environmental situations in which bioaccumulation processes occur is prerequisite to the selection of suitable laboratory test systems, the results of which can reliably be used to evaluate the bioaccumulation potential of a chemical in the environment.

\section{BIOACCUMULATION IN THE AQUATIC ENVIRONMENT}

6. Two experimental approaches are in use to study bioaccumulation in the aquatic organisms: integrated test systems (ecosystems, food chains) and compartmentalised test systems (individual organism or steps of food chains). Both approaches clearly disclose an existing bioaccumulation potential of a chemical. However, the latter approach gives rise to less problems regarding handling, reproducibility of the absolute data measured and the elucidation of the individual factors responsible for the bioaccumulation process.

7. The Expert Group considered differences between testing with flow-through and static systems. The concentration of a chemical at steady-state conditions in a static system is normally lower than the initial concentration. Under extreme conditions, this may cause analytical problems, and lead to measurements under dynamic conditions. 
8. With respect to direct bioaccumulation in individual species, there is a clear correlation between bioaccumulation and lipophilicity [expressed as partition coefficient (n-octanol/water) $=\mathrm{P}_{\text {ow }}$ ] of nonionised organic chemicals. Although exceptions are known, they normally relate to cases where $\mathrm{P}_{\mathrm{ow}}$ over estimates the bioconcentration factor (BCF). Therefore, the partition coefficient, $\mathrm{P}_{\mathrm{ow}}$, may be regarded as a good, conservative predictor of bioaccumulation of these chemicals. The more lipophilic a chemical is (i.e. the higher its $\mathrm{P}_{\mathrm{ow}}$ ), the slower is it desorbed, i.e. depurated from the organism. Depuration cannot always be described by first-order kinetics. Therefore, apparent half-life times should not be extrapolated beyond the measured range.

9. There might be cases where reaction of a chemical in a living organism may result in derivatives which are more lipophilic than the parent chemical, or in binding of the chemical, or parts of it, to cell constituents (e.g. inorganic mercury $\rightarrow$ methylmercury $\rightarrow$ reaction product with cell constituents). In the former case, bioaccumulation of the derivative will be higher than expected from the $\mathrm{P}_{\mathrm{ow}}$ of the parent chemical. In the latter case, the retention of the bound chemical will depend upon the stability of the binding and the metabolic half-life of the cell constituent to which the chemical is bound. Candidates for such a behaviour may be found among inorganic and metal-organic chemicals. Certain chemicals which ionise under physiological conditions also may be considered as candidates if the impact of complex and ion-pair formation upon bioaccumulation cannot be evaluated. In this case, $\mathrm{P}_{\mathrm{ow}}$ is not a good predictor of the bioaccumulation potential.

10. In fish it has been demonstrated that the lipid content has decisive influence on the magnitude of the BCF. Other sources of variation can probably be reduced in importance by a stricter harmonization of test conditions (e.g. ratio of biomass/water volume, ratio of biomass/concentration of chemical, timing of sampling, feeding of test fish, etc.).

11. Interpretation of different BCFs found for a given compound with different fish species might be facilitated by relating a measured value to that of a reference compound of known stability and comparable bioaccumulation behaviour (e.g. the ratio: $\mathrm{BCF}$ test chemical/BCF reference, i.e. bioaccumulation index). Laboratory intercomparison was carried out to ascertain the applicability and validity of the five recommended fish accumulation tests.

\section{TERRESTRIAL ENVIRONMENT}

12. Uptake of chemicals by terrestrial animals is mainly by food. The dermal route might contribute to this uptake to some extent in animals inhabiting soil. Significant uptake by the respiratory route would be rather an exception. Consequently, species at the top of food chains are expected to show the highest concentrations of chemicals that exhibit bioaccumulation potential and enter such chains at any lower level.

13. In mammals and birds, it has been shown that under constant uptake conditions, a dynamic equilibrium is also reached as manifested by a constant concentration of the chemical in particular organs and tissues of the test animals (apparent plateau). The rate with which this equilibrium is reached, and its level, are characteristics of a given chemical and test species, and of the chemical's concentration in food. The same holds true for the rate of depuration/excretion which is observed after discontinuation of the exposure.

14. Using, as measure, the ratio of concentrations in the respective tissue at plateau and in the food, i.e. the $\mathrm{BCF}$, these studies demonstrated that the bioaccumulation factor, $\mathrm{BCF}$, of chemicals in terrestrial animals is of an order of magnitude lower than that found in aquatic animals, such as fishes. 
15. Studies on mammals and birds have confirmed the experience gathered with aquatic organisms: the lipophilicity of a chemical of sufficient metabolic stability is the property which essentially determines the degree of bioaccumulation. Consequently, the n-octanol/water partition coefficient is also a good predictor of the potential of a non-ionised organic chemical to bioaccumulate in lipid tissues of terrestrial animals.

16. With plants, the potential for significant exposure to organic chemicals are taken up, they generally do not bioaccumulate. Therefore, bioaccumulation testing in plants is not indicated.

\section{TEST PHILOSOPHY IN BIOACCUMULATION TESTING}

17. Testing of the bioaccumulation potential of a given chemical should proceed in steps, with increases in the extent and degree of information furnished and in the experimental efforts required. This step sequence approach should coincide with the approaches selected in biodegradation and ecotoxicity testing, to allow optimum harmonisation of the total testing programme.

18. The decision not to test a non-ionised organic chemical will be based upon the simultaneous assessment of its relevant physical-chemical properties and its degradability. For instance, if a chemical is readily biodegradable, as defined above under "Biodegradation in Water", then no bioaccumulation testing is required. Biodegradation often results in products which have partially or completely lost the original bioaccumulation potential.

19. For inorganic and metallo-organic compounds the bioaccumulation potential cannot unequivocally be established by the n-octanol/water partitioning test. Such compounds should undergo a bioaccumulation test on fish which, at this screening stage, would be a static test.

20. For organic compounds that ionise under physiological conditions ( $\mathrm{pH} 3-9$ ), the use of noctanol/water partition coefficient is only predictive of bioaccumulation if evidence exists that the chemical will show no reactivity to cell constituents.

21. If this evidence cannot be provided, a bioaccumulation test with fish should be performed for the patent compound, or its products of rapid transformation, unless the compound is known to be readily degradable. At the screening stage, a static test might be sufficient if applicable.

\section{BEHAVIOUR IN SOILS AND SEDIMENTS}

22. The study of the fate of chemicals in soils or sediments is of particular importance since contamination can be long lasting and difficult to reverse due to limited opportunity for mobilisation and dilution.

23. When performing tests for biodegradation, or the adsorption coefficient, normally, the structure of the soil is destroyed, for example by sieving or washing while the texture is maintained. This is of minor importance for the determination of the degradability potential.

24. However, for those exceptional cases, where, ultimately, a realistic environment model study is indicated, it is essential to include the soil and sediment in a test system so that their structure remains functionally undisturbed, e.g. by using soil monoliths of field experiments.

25. Detailed information should - where feasible - be provided on special soil properties (e.g. texture, clay-content, organic carbon content, etc.). These specifications vary depending on the aim of the test. The use of specific soil types is recommended in order to ensure wide acceptance of the results. 
26. For soil characterisation the Expert Group recommended the use of the American soil classification scheme (Soil Survey Staff and Collaborators, 1967). In developing a guideline for adsorption testing, the Group identified three soil types. Since these soil types offer a range of opportunities, they are also recommended for use in biodegradation and/or leaching studies. They provide sufficient flexibility and vary significantly in, for example, cation exchange capacity, clay content, organic matter content, exchangeable cautions and $\mathrm{pH}$.

27. In summary, they can be described as follows:

(1) very strongly to strongly acid sandy soil, e.g. Spodosol;

(2) moderately or slightly acid loamy soil, e.g. Alfisol;

(3) neutral to slightly alkaline loamy soil, e.g. Entisol.

All three are common in temperate zones but will not be representative of arid or tropical regions.

28. The soil should be characterised by the following parameters: $\mathrm{pH}$, clay content, redox potential (Eh), organic matter, nutrient status $(\mathrm{N}, \mathrm{P}, \mathrm{K})$. In case of column studies, pore volume should be determined. It is recommended that a control chemical be used as a regular procedure in each experiment as a check on the microbial activity of the soil.

29. The Expert Group felt that water logged soils flushed with inert gas may be used as an acceptable substitute for sediments with anaerobic conditions. Care should be taken after water logging to allow an adequate period for the acclimatisation of the microflora and the stabilisation of the redox potential. 